\title{
A case of highly aggressive anaplastic seminoma of the testis presenting as fungating scrotal lesion
}

\author{
Massimiliano Creta ${ }^{1}$, Vincenzo Mirone ${ }^{2}$, Sergio Di Meo ${ }^{1}$, Roberto Buonopane ${ }^{1}$, Nicola Longo ${ }^{2}$, \\ Ferdinando Fusco ${ }^{2}$, Nicola Rosario Forte ${ }^{3}$, Vittorio Imperatore ${ }^{1}$ \\ ${ }^{1}$ Unità Operativa di Urologia, Ospedale Buon Consiglio - Fatebenefratelli, Napoli, Italy; \\ ${ }^{2}$ Clinica Urologica, Università Federico II di Napoli, Napoli, Italy; \\ ${ }^{3}$ Unità Operativa di Anatomia Patologica, Ospedale Fatebenefratelli, Benevento, Italy.
}

\begin{abstract}
Summary Anaplastic seminoma (AS) is an uncommon histological variant of classical seminoma of the testis and account for 5\%-15\% of cases. It is poorly described in the scientific literature. We present the case of a 50-years-old homeless man presenting with fever, marked left scrotal hardness and a fungating left scrotal lesion. He underwent left orchiopexy 40 years before. A computed tomography with contrast media showed a suspect testis cancer with scrotal involvment, extensive intralesional necrosis and multiple systemic metastases. A wide excision of the left hemiscrotum including the testis was performed in order to prevent severe local and systemic infectious complications. Histological examination revealed an AS. General conditions showed a rapid deterioration and the patient died on post operative day 10.
\end{abstract}

KEY WORDS: Anaplastic seminoma; Cryptorchidism;

Fungating lesion.

Submitted 1 May 2017; Accepted 15 May 2017

\section{INTRODUCTION}

Testis cancer (TC) represents $1 \%$ of male neoplasms and $5 \%$ of urological tumours in western society (1). The predominant histology is germ cell tumour (GCT) and peak incidence is in the third decade of life for non-seminoma, and in the fourth decade for pure seminoma (1). To date, most cases of TC are diagnosed at an early stage, are readily treatable and consequently exhibit an excellent prognosis (1). The anaplastic variant of classical seminoma has overall morphologic features of seminoma but with higher number of mitotic figures per high powered field, cellular irregularity, no fibrovascular septae, few lymphoctes, focal necrosis, and pleomorphic cells with nonclear cytoplasm (2). Anaplastic seminoma (AS) constitutes $5 \%-15 \%$ of testis seminomas and, to date, there are conflicting reports about the clinical behaviour of this neoplasm (2). Due to the low number of cases mentioned in scientific literature it is difficult to determine whether the anaplastic differentiation predicts bad prognosis, like other solid tumors with anaplastic biology. In this report, we describe a case of a metastatic AS presenting as a fungating scrotal lesion with infectious complications in a patient with history of previous orchidopexy.

\section{Case report}

A 50-years-old homeless man was referred to emergency department for left scrotal tumescence, pain, fever, and fetid discharge through the scrotal skin. His past surgical history was relevant for left orchidopexy performed at the age of 10. Physical examination revealed an enlarged and hard left hemiscrotum with a cutaneous fungatng lesion characterized by purulent discharge and multiple cutaneous nodules on the abdomen and thorax (Figure 1). Baseline levels of serum alfa fetoprotein and beta subunit of human chorionic gonadotropin were 2.26 $\mathrm{ng} / \mathrm{mL}$ and $113.8 \mathrm{mUI} / \mathrm{mL}$, respectively. White blood cells count was $15.000 / \mathrm{mm}^{3}$ and C-reactive protein was $202 \mathrm{mg} / \mathrm{L}$. A whole body contrast enhanced computed tomography (CT) showed an enhancing solid mass (10 $x$

Figure 1.

A) Clinical picture showing a fungating cutaneous neoplastic ulcers at the level of the left hemiscrotum and the surgical scar of previous orchidopexy (arrowhead).

B) Contrast enhanced CT scan showing a large enhancing solid mass $(10 \times 7 \mathrm{~cm})$ with regional extension to the skin in the left hemiscrotum and a large area of inratumoral coagulative necrosis (asterisk).

C) Contrast enhanced CT scan showing a cutaneous metastasis at the level of the anterior right abdominal wall. D) Contrast enhanced CT scan showing liver, renal, nodal and bone metastases.
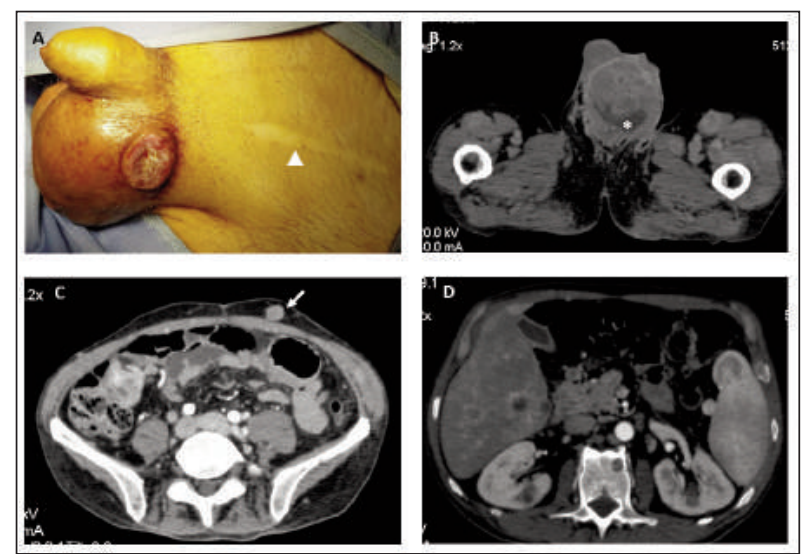

No conflict of interest declared. 
$7 \mathrm{~cm}$ ) in the left hemiscrotum with regional extension to the skin and characterized by a large area of intralesional coagulative necrosis. Multiple cutaneous, liver, lung, adrenal, kidney, nodal and bone metastases were also evident. Fever, leukocytosis and elevated C-reactive protein levels persisted despite antimicrobial therapy. Surgery was consequently planned and a wide excision of the left hemiscrotum including the testis was carried out in order to avoid the occurrence of a severe necrotizing fasciitis.

Histopathological examination showed a poorly differentiated testis neoplasm characterized by wide areas of necrosis and hemorrage, as well as by sarcomatoid and squamous features compatible with AS invading scrotal skin. Patient's general conditions showed a rapid deterioration and he died on post operative day 10 due to acute respiratory failure.

\section{Discussion}

To date, there are very few studies and limited information about AS in the scientific literature and the clinical behaviour of this histological variant is a matter of debate (2). Herein, we present the case of a patient with AS arising in a cryptorchid testis 40 years after orchiopexy and characterized by an highly aggressive clinical behaviour. This patient was a unique case due to multiple features and, to our knowledge, there are no other similar reports in the literature. About 10\% of testis GCT occur in men with a history of cryptorchidism and the hystologic subtype most commonly associated with cryptorchidism is seminoma. According to some evidences, there is a direct correlation between how long the testis is subjected to a cryptorchid position and the incidence of cancer. Consequently, contemporary guidelines recommed surgical correction of cryptorchidism before the age of 12 months or 18 months the latest (3). Moreover, a careful surveillance for malignant changes is advocated postorchiopexy (3). To our knowledge, there are very few cases of AS arising in a cryptorchid testis submitted to orchidopexy and this case also underlines the importance of long-term follow-up of patients after orchidopexy. Skin invasion and cutaneous metastases are uncommon in patients with TC and these features have been associated with a poor prognosis. In the present case, the lack of a careful surveillance and the delayed diagnosis due to the poor socioeconomic status also contributed to the poor prognosis. Every patient with a suspected testicular mass must undergo inguinal exploration (1). In cases of life threatening disseminated disease, lifesaving chemotherapy should be given up-front, especially when the clinical picture is very likely TC and/or tumour markers are increased. Orchiectomy may be delayed until clinical stabilisation occurs (1).

Treatment of AS should be the same as for classical seminoma, stage for stage (2). Necrotic neoplastic tissues, like biologically inhert bodies, may represent a substrate for infection and abscess formation (4). Urgent surgery was planned in this case due to the evidence of tumor necrosis, local infectious complications, fever and leukocytosis, a clinical picture that may represent the prelude of a lifethreatening necrotizing fascitis and demands prompt and aggressive treatment. However, in the light of the clinical course of present case, the role of any surgical approach in similar patients should be carefully discussed.

\section{Conclusions}

AS represents an uncommon variant of testis seminoma that may occur in a cryptorchid testis even many years after orchidopexy and present with highly aggressive clinical features.

\section{RefEREnCES}

1. Albers P, Albrecht W, Algaba F, et al. European Association of Urology.Guidelines on Testicular Cancer: 2015 Update. Eur Urol. $201 ; 68: 1054-68$.

2. Stein ME, Zidan J, Charas T, et al. Anaplastic variant of classical seminoma of the testis: northern Israel oncology center experience and brief review of literature. Rambam Maimonides Med J. 2014; 5:e0006.

3. Tekgul S, Riedmiller H, Dogan HS, et al. Guidelines on paediatric urology. Arnhem, The Netherlands: European Association of Urology, European Society for Paediatric Urology. 2013.

4. Cennamo P, Montuori N, Trojsi G, et al. Biofilms in churches built in grottoes. Sci Total Environ. 2016; 543:727-38.

\author{
Correspondence \\ Massimiliano Creta, MD (Corresponding author) \\ max.creta@gmail.com \\ Sergio Di Meo, MD \\ s.dimeo72@gmail.com \\ Roberto Buonopane, MD \\ robertobuonopane@libero.it \\ Vittorio Imperatore, $\mathrm{MD}$ \\ v.imperatore@alice.it \\ Unità Operativa di Urologia, \\ Buon Consiglio Fatebenefratelli Hospital, Napoli \\ Via A. Manzoni, 220 - 80123 Napoli, Italy \\ Vincenzo Mirone, MD, Professor of Urology \\ Nicola Longo, MD \\ Ferdinando Fusco, MD \\ Clinica Urologica, Università Federico II di Napoli \\ Via S. Pansini, 5 - 80131 Napoli, Italy \\ Nicola Rosario Forte, MD \\ Unità Operativa di Anatomia Patologica, \\ Ospedale Fatebenefratelli, Benevento \\ Viale Principe di Napoli, 14 - 82100 Benevento, Italy
}

